\title{
Remotest Points and Best Proximity Points in Metric Spaces
}

\author{
M. Ahmadi. Baseri ${ }^{*}$ H. Mazaheri \\ Department of Mathematics, Yazd University, Yazd, Iran
}

Received February 28, 2019; Revised April 12, 2019; Accepted April 21, 2019

Copyright (C)2019 by authors, all rights reserved. Authors agree that this article remains permanently open access under the terms of the Creative Commons Attribution License 4.0 International License

\begin{abstract}
In this paper, we give sufficient conditions for the existence of remotest points with recurrence relations. Then, we apply these relations to generalize recent best proximity point theorems. The findings of the present research contribute and enrich previous results reviewed in the literature.
\end{abstract}

Keywords Recurrence Relations, Remotest points, Property $U C_{f}$, Property $U C$, Best Proximity Points MSC (2010): 41A65, 41A52, 46N10.

\section{Introduction}

Let $A$ and $B$ be nonempty bounded subsets of a metric space $(X, d)$. The set $A$ is said to be remotest from a point $x \in X$, if there exists a point $x_{0} \in A$ such that $\delta(x, A)=\sup \{d(x, y): y \in A\}=d\left(x, x_{0}\right)$. The point $x_{0}$ is called a remotest point of $A$ from $x$. If there is a pair $\left(x_{0}, y_{0}\right) \in A \times B$ for which $d\left(x_{0}, y_{0}\right)=\delta(A, B)$, that $\delta(A, B)$ is remotest distance of $A$ and $B$, define by

$$
\delta(A, B)=\sup \{d(x, y): x \in A, y \in B\} .
$$

Then the pair $\left(x_{0}, y_{0}\right)$ is called a remotest pair for $A$ and $B$ and put

$$
F(A, B)=\{(x, y) \in A \times B: d(x, y)=\delta(A, B)\}
$$

as the set of all remotest pairs $(A, B)$.

The problem of characterizing remotest sets has applications in the study of approximation theory and geometry of Banach spaces, see $[5,6,13]$. One could find more results about remotest points in $[9,11,12]$.

In the following this research, in 2015, we introduced the concept of remotest points for a cyclic map $T: A \cup B \rightarrow A \cup B$ i.e. $T(A) \subseteq B$ and $T(B) \subseteq A$. As follows.

Definition 1.1 [2] Let $A$ and $B$ be nonempty bounded subsets of a metric space $X$ and $T: A \cup B \rightarrow A \cup B$ be a cyclic map. The point $x \in A \cup B$ is a remotest point of the map $T$, if $d(x, T x)=\delta(A, B)$.

On the other hand, the study of existence of best proximity points for cyclic contraction mappings is very interesting. More precisely, for two given nonempty subsets $A$ and $B$ of a metric space $(X, d)$, a point $x \in A \cup B$ is called a best proximity point of cyclic map $T: A \cup B \rightarrow A \cup B$, if $d(x, T x)=d(A, B)$ where $d(A, B)=\inf \{d(a, b): a \in A, b \in B\}$. The first result in this area was reported by Kirk and et al [10]. Later, many authors continued investigation and more results have been obtained, such as, $[1,3,4,7,8]$.

In this paper, we first present recurrence relation as follows, $T: A \cup B \rightarrow A \cup B$ is a continuous cyclic contraction map, assuming $x_{0} \in A$, define $x_{n+1}=T x_{n}$ for every $n \in \mathbb{N} \cup\{0\}$ and let $d_{n}=d\left(x_{n}, x_{n+1}\right)$. Then we find remotest points of the continuous cyclic map $T$, with recurrence relations. Finally, we give best proximity point results by using this relation. To prove our results in section 3 , we recall following definition. 
Definition 1.2 [14] Let $A$ and $B$ be nonempty bounded subsets of a metric space $X$. Then $(A, B)$ is said to satisfy the property $U C$ if the following holds:

If $\left\{x_{n}\right\}$ and $\left\{z_{n}\right\}$ are sequences in $A$ and $\left\{y_{n}\right\}$ is a sequence in $B$ such that $\lim _{n \rightarrow \infty} d\left(x_{n}, y_{n}\right)=d(A, B)$ and $\lim _{n \rightarrow \infty} d\left(z_{n}, y_{n}\right)=$ $d(A, B)$, then $d\left(x_{n}, z_{n}\right) \rightarrow 0$ holds.

\section{Remotest Points}

We prove the following result which will be needed in what follows.

Proposition 2.1 Let $A$ and $B$ be nonempty bounded subsets of a metric space $X$ and

$$
d_{n+k} \geq \alpha^{k} d_{n}+\left(1-\alpha^{k}\right) \delta(A, B)
$$

where $\alpha, k$ are constant such that $0<\alpha<1$ and $k \in \mathbb{N}$. Then $d_{n+k} \rightarrow \delta(A, B)$ as $n \rightarrow \infty$.

Proof. Let $m \in \mathbb{N}$. By inequality (2.1),

$d_{k m+k-1} \geq \alpha^{k m} d_{k-1}+\left(1-\alpha^{k}\right)\left(\sum_{i=0}^{m-1} \alpha^{k i}\right) \delta(A, B)$,

$d_{k m+k-2} \geq \alpha^{k m} d_{k-2}+\left(1-\alpha^{k}\right)\left(\sum_{i=0}^{m-1} \alpha^{k i}\right) \delta(A, B)$,

$d_{k m+k-3} \geq \alpha^{k m} d_{k-3}+\left(1-\alpha^{k}\right)\left(\sum_{i=0}^{m-1} \alpha^{k i}\right) \delta(A, B)$,

continuing this process,

$d_{k m+1} \geq \alpha^{k m} d_{1}+\left(1-\alpha^{k}\right)\left(\sum_{i=0}^{m-1} \alpha^{k i}\right) \delta(A, B)$ and

$d_{k m} \geq \alpha^{k m} d_{0}+\left(1-\alpha^{k}\right)\left(\sum_{i=0}^{m-1} \alpha^{k i}\right) \delta(A, B)$.

Letting $m \rightarrow \infty, \lim _{m \rightarrow \infty} d_{k m+i} \geq \delta(A, B), \quad i=0,1, \ldots, k-1$. On the other hand, $\delta(A, B) \geq \lim _{m \rightarrow \infty} d_{k m+i}, \quad i=$ $0,1, \ldots, k-1$. So

$$
\lim _{m \rightarrow \infty} d_{k m+i}=\delta(A, B), \quad i=0,1, \ldots, k-1 .
$$

Therefore $d_{n+k} \rightarrow \delta(A, B)$ as $n \rightarrow \infty$.

In following theorem give remotest point when one of the sets is boundedly compact.

Theorem 2.1 Let $A$ and $B$ be nonempty bounded subsets of a metric space $X$ and relation (2.1) holds. If either $A$ or $B$ is boundedly compact, then there exists $x \in A \cup B$ with $d(x, T x)=\delta(A, B)$.

Proof. Let $k$ be an even positive integer. So $x_{2 n+k} \in A$. Suppose $A$ is boundedly compact. Since the sequence $\left\{x_{2 n+k}\right\}$ is bounded and $A$ is boundedly compact. Hence $\left\{x_{2 n+k}\right\}$ has a convergent subsequence in $A$. Let $\left\{x_{2 n_{l}+k}\right\}$ be a subsequence of $\left\{x_{2 n+k}\right\}$ with $x_{2 n_{l}+k} \rightarrow x \in A$ as $l \rightarrow \infty$. Since $T$ is a continuous map and $d\left(x_{2 n_{l}+k}, T x_{2 n_{l}+k}\right) \rightarrow d(A, B)$ as $l \rightarrow \infty$. Therefore $d(x, T x)=\delta(A, B)$. The proof when $k$ is an odd positive integer and $B$ is boundedly compact is similar.

Example 2.1 Given $0<\alpha<1, k \in \mathbb{N}$. let $A$ and $B$ be nonempty bounded subsets of $l^{p}, 1 \leq p<\infty$ where $p$ is odd number, defined by $A=\left\{\left(\left(1-\alpha^{2 n}\right) e_{2 n}\right): n \in \mathbb{N}\right\}$ and $B=\left\{\left(\left(1-\alpha^{2 m-1}\right) e_{2 m-1}\right): m \in \mathbb{N}\right\}$. Suppose

$$
T\left(\left(1-\alpha^{2 n}\right) e_{2 n}\right)=\left(1-\alpha^{2 n+1}\right) e_{2 n+1}
$$

and

$$
T\left(\left(1-\alpha^{2 m-1}\right) e_{2 m-1}\right)=\left(1-\alpha^{2 m}\right) e_{2 m}
$$

Then

$$
d_{n+k} \geq \alpha^{k} d_{n}+\left(1-\alpha^{k}\right) \delta(A, B),
$$

where $\alpha, k$ are constant such that $0<\alpha<1$ and $k \in \mathbb{N}$.

Proof. Here $\delta(A, B)=2^{1 / p}$.

$$
\begin{aligned}
\alpha^{k}\left(\left(1-\alpha^{2 m}\right)^{p}+\left(1-\alpha^{2 n+1}\right)^{p}\right)^{1 / p}+\left(1-\alpha^{k}\right) 2^{1 / p} & =\left(\left(1+\alpha^{k}-1-\alpha^{2 m+k}\right)^{p}\right. \\
& \left.+\left(1+\alpha^{k}-1-\alpha^{2 n+1+k}\right)^{p}\right)^{1 / p} \\
& +\left(1-\alpha^{k}\right) 2^{1 / p} \\
& \leq\left(\left(1-\alpha^{2 m+k}\right)^{p}+\left(1-\alpha^{2 n+1+k}\right)^{p}\right)^{1 / p} \\
& -2^{1 / p}\left(1-\alpha^{k}\right) \\
& +\left(1-\alpha^{k}\right) 2^{1 / p} \\
& =\left(\left(1-\alpha^{2 m+k}\right)^{p}\right. \\
& \left.+\left(1-\alpha^{2 n+1+k}\right)^{p}\right)^{1 / p}
\end{aligned}
$$


Which implies that

$$
d_{n+k} \geq \alpha^{k} d_{n}+\left(1-\alpha^{k}\right) 2^{1 / p}
$$

Definition 2.1 Let $A$ and $B$ be nonempty bounded subsets of a metric space $X$. Then $(A, B)$ is said to satisfy the property $U C_{f}$ if the following holds:

If $\left\{x_{n}\right\}$ and $\left\{z_{n}\right\}$ are sequences in $A$ and $\left\{y_{n}\right\}$ is a sequence in $B$ such that $\lim _{n \rightarrow \infty} d\left(x_{n}, y_{n}\right)=\delta(A, B)$ and $\lim _{n \rightarrow \infty} d\left(z_{n}, y_{n}\right)=$ $\delta(A, B)$, then $d\left(x_{n}, z_{n}\right) \rightarrow 0$ holds.

Theorem 2.2 Let $A$ and $B$ be nonempty closed and bounded subsets of a complete metric space $X$ such that (A,B) has a property $U C_{f}$ and relation (2.1) holds. Then $T$ has a remotest point.

Proof. We show that for each $\epsilon>0$, there exists a positive integer $N_{0}$ such that for all $m>n \geq N_{0}$,

$$
d\left(x_{2 m+k}, x_{2 n+k+1}\right)>\delta(A, B)-\epsilon .
$$

On the contrary, assume that there exists $\epsilon_{0}>0$ such that for each $l \geq 1$, there is $m_{l}>n_{l} \geq l$ satisfying

$$
d\left(x_{2 m_{l}+k}, x_{2 n_{l}+k+1}\right) \leq \delta(A, B)-\epsilon_{0}
$$

and

$$
d\left(x_{2 m_{l}-2+k}, x_{2 n_{l}+k+1}\right)>\delta(A, B)-\epsilon_{0} .
$$

It follows from (2.3) and (2.4) that

$$
\begin{aligned}
d\left(x_{2 m_{l}-2+k}, x_{2 n_{l}+k+1}\right)-d\left(x_{2 m_{l}+k}, x_{2 m_{l}-2+k}\right) & \leq d\left(x_{2 m_{l}+k}, x_{2 n_{l}+k+1}\right) \\
& \leq \delta(A, B)-\epsilon_{0} .
\end{aligned}
$$

Letting $l \rightarrow \infty$, property $U C_{f}$ implies

$$
\lim _{k \rightarrow \infty} d\left(x_{2 m_{l}+k}, x_{2 n_{l}+k+1}\right)=\delta(A, B)-\epsilon_{0} .
$$

By relation (2.1),

$$
\begin{aligned}
\delta(A, B)-\epsilon_{0} & \geq d\left(x_{2 m_{l}+2 k}, x_{2 n_{l}+2 k+1}\right) \\
& \geq \alpha^{k} d\left(x_{2 m_{l}+k}, x_{2 n_{l}+k+1}\right)+\left(1-\alpha^{k}\right) \delta(A, B)
\end{aligned}
$$

Letting $l \rightarrow \infty$ and using (2.5), we obtain

$$
\begin{aligned}
\delta(A, B)-\epsilon_{0} & \geq \alpha^{k}\left(\delta(A, B)-\epsilon_{0}\right)+\left(1-\alpha^{k}\right) \delta(A, B) \\
& =\delta(A, B)-\alpha^{k} \epsilon_{0}
\end{aligned}
$$

which is a contradiction. Therefore by (2.1) and property $U C_{f},\left\{x_{2 n+k}\right\}$ is a Cauchy sequence by property $U C_{f}$. Now the completeness of $X$ and the closedness of $A$ imply that $\lim _{n \rightarrow \infty} x_{2 n+k}=z \in A$, whenever $k$ is a even number. (if $k$ is a odd number, $x_{2 n+k} \rightarrow z \in B$ as $n \rightarrow \infty$.) Since $T$ is a continuous map and $d\left(x_{2 n+k}, x_{2 n+k+1}\right) \rightarrow \delta(A, B)$ as $n \rightarrow \infty$, hence $d(z, T z)=\delta(A, B)$. Therefore, $T$ has a remotest point.

\section{Best Proximity Points}

First, we give a useful approximation result.

Proposition 3.1 Let $A$ and $B$ be nonempty subsets of a metric space $X$ and

$$
d_{n+k} \leq \alpha^{k} d_{n}+\left(1-\alpha^{k}\right) d(A, B)
$$

where $\alpha, k$ are constant such that $0<\alpha<1, k \in \mathbb{N}$. Then $d_{n+k} \rightarrow d(A, B)$ as $n \rightarrow \infty$.

Proof. Let $m \in \mathbb{N}$ By inequality (3.1), 
$d_{k m+k-1} \leq \alpha^{k m} d_{k-1}+\left(1-\alpha^{k m}\right) d(A, B)$,

$d_{k m+k-2} \leq \alpha^{k m} d_{k-2}+\left(1-\alpha^{k m}\right) d(A, B)$,

$d_{k m+k-3} \leq \alpha^{k m} d_{k-3}+\left(1-\alpha^{k m}\right) d(A, B)$,

continuing this process,

$d_{k m+1} \leq \alpha^{k m} d_{1}+\left(1-\alpha^{k m}\right) d(A, B)$ and

$d_{k m} \leq \alpha^{k m} d_{0}+\left(1-\alpha^{k m}\right) d(A, B)$,

Letting $m \rightarrow \infty$,

$$
d_{k m+i} \rightarrow d(A, B), \quad i=0,1, \ldots, k-1 .
$$

So $d_{n+k} \rightarrow d(A, B)$ as $n \rightarrow \infty$.

Next, we are ready to state our main results in this section.

Proposition 3.2 Let $A$ and $B$ be nonempty subsets of a metric space $X$ and relation (3.1) holds. Then the sequences $\left\{x_{2 n+k}\right\}$ and $\left\{x_{2 n+k+1}\right\}$ are bounded.

Proof. Since the sequence $\left\{d\left(x_{2 n+k}, x_{2 n+k+1}\right)\right\}$ converges to $d(A, B)$ as $n \rightarrow \infty$, it is enough to prove that $\left\{x_{2 n+k+1}\right\}$ is bounded. Suppose $\left\{x_{2 n+k+1}\right\}$ is not bounded, then there exists a $N_{0}$ such that

$$
d\left(x_{1+k}, x_{2 N_{0}+k+1}\right)>M
$$

and

$$
d\left(x_{1+k}, x_{2 N_{0}+1}\right) \leq M
$$

where $M>\max \left\{\frac{\alpha^{k}}{1-\alpha^{k}} d\left(x_{1}, x_{1+k}\right)+d(A, B), d\left(x_{1+k}, x_{1+2 k}\right\}\right.$. By relation (3.1),

$$
\begin{aligned}
M & <d\left(x_{1+k}, x_{2 N_{0}+k+1}\right) \\
& \leq \alpha^{k} d\left(x_{1}, x_{2 N_{0}+1}\right)+\left(1-\alpha^{k}\right) d(A, B) \\
& \leq \alpha^{k} d\left(x_{1}, x_{1+k}\right)+\alpha^{k} d\left(x_{1+k}, x_{2 N_{0}+1}\right)+\left(1-\alpha^{k}\right) d(A, B) \\
& \leq \alpha^{k} d\left(x_{1}, x_{1+k}\right)+\alpha^{k} M+\left(1-\alpha^{k}\right) d(A, B)
\end{aligned}
$$

Thus $M<\frac{\alpha^{k}}{1-\alpha^{k}} d\left(x_{1}, x_{1+k}\right)+d(A, B)$, which is a contradiction.

The following Theorem explain a result when one of the sets is boundedly compact.

Theorem 3.1 Let $A$ and $B$ be nonempty subsets of a metric space $X$ and relation (3.1) holds. If either $A$ or $B$ is boundedly compact, then there exists $x \in A \cup B$ with $d(x, T x)=d(A, B)$.

Proof. Let $k$ be even number. So $x_{2 n+k} \in A$. Suppose $A$ is boundedly compact. Since the sequence $\left\{x_{2 n+k}\right\}$ is bounded and $A$ is boundedly compact. Hence $\left\{x_{2 n+k}\right\}$ has a convergent subsequence in $A$. Let $\left\{x_{2 n_{l}+k}\right\}$ be a subsequence of $\left\{x_{2 n+k}\right\}$ with $x_{2 n_{l}+k} \rightarrow x \in A$ as $l \rightarrow \infty$. Since $T$ is a continuous map and $d\left(x_{2 n_{l}+k}, T x_{2 n_{l}+k}\right) \rightarrow d(A, B)$ as $l \rightarrow \infty$. Therefore $d(x, T x)=d(A, B)$. The proof when $k$ is odd number and $B$ is boundedly compact is similar.

Example 3.1 Given $0<\alpha<1, k \in \mathbb{N}$. let $A$ and $B$ be nonempty subsets of $l^{P}, 1 \leq p \leq \infty$ defined by $A=\left\{\left(\left(1+\alpha^{2 n}\right) e_{2 n}\right)\right.$ : $n \in \mathbb{N}\}$ and $B=\left\{\left(\left(1+\alpha^{2 m-1}\right) e_{2 m-1}\right): m \in \mathbb{N}\right\}$. Suppose

$$
T\left(\left(1+\alpha^{2 n}\right) e_{2 n}\right)=\left(1+\alpha^{2 n+1}\right) e_{2 n+1}
$$

and

Then relation (2.1) holds.

$$
T\left(\left(1+\alpha^{2 m-1}\right) e_{2 m-1}\right)=\left(1+\alpha^{2 m}\right) e_{2 m}
$$

Proof. The case when $p=\infty$ is easy to check, so we consider $1 \leq p<\infty$.

$$
\begin{aligned}
\left(\left(1+\alpha^{2 m+k}\right)^{p}+\left(1+\alpha^{2 n+1+k}\right)^{p}\right)^{1 / p} & =\left(\left(1+\alpha^{k}-\alpha^{k}+\alpha^{2 m+k}\right)^{p}\right. \\
& \left.+\left(1+\alpha^{k}-\alpha^{k}+\alpha^{2 n+1+k}\right)^{p}\right)^{1 / p} \\
& \leq\left(\left(\alpha^{k}+\alpha^{2 m+k}\right)^{p}+\left(\alpha^{k}+\alpha^{2 n+1+k}\right)^{p}\right)^{1 / p} \\
& +2^{1 / p}\left(1-\alpha^{k}\right) \\
& \leq \alpha^{k}\left(\left(1+\alpha^{2 m}\right)^{p}+\left(1+\alpha^{2 n+1}\right)^{p}\right)^{1 / p} \\
& +2^{1 / p}\left(1-\alpha^{k}\right) .
\end{aligned}
$$

Which implies that

where $d(A, B)=2^{1 / p}$.

$$
d_{n+k} \leq \alpha^{k} d_{n}+\left(1-\alpha^{k}\right) 2^{1 / p}
$$


Theorem 3.2 Let $A$ and $B$ be nonempty closed subsets of a complete metric space $X$ such that $(A, B)$ has a property $U C$ and relation (3.1) holds. Then $T$ has a best proximity point.

Proof. We show that for each $\epsilon>0$, there exists a positive integer $N_{0}$ such that for all $m>n \geq N_{0}$,

$$
d\left(x_{2 m+k}, x_{2 n+k+1}\right)<d(A, B)+\epsilon .
$$

On the contrary, assume that there exists a $\epsilon_{0}>0$ such that for each $l \geq 1$, there is $m_{l}>n_{l} \geq l$ satisfying

$$
d\left(x_{2 m_{l}+k}, x_{2 n_{l}+k+1}\right) \geq d(A, B)+\epsilon_{0}
$$

and

$$
d\left(x_{2 m_{l}-2+k}, x_{2 n_{l}+k+1}\right)<d(A, B)+\epsilon_{0} .
$$

It follows from (3.3) and (3.4) that

$$
\begin{aligned}
d(A, B)+\epsilon_{0} & \leq d\left(x_{2 m_{l}+k}, x_{2 n_{l}+k+1}\right) \\
& \leq d\left(x_{2 m_{l}+k}, x_{2 m_{l}-2+k}\right)+d\left(x_{2 m_{l}-2+k}, x_{2 n_{l}+k+1}\right) \\
& <d\left(x_{2 m_{l}+k}, x_{2 m_{l}-2+k}\right)+d(A, B)+\epsilon_{0} .
\end{aligned}
$$

Letting $l \rightarrow \infty$, and with property UC implies

$$
\lim _{k \rightarrow \infty} d\left(x_{2 m_{l}+k}, x_{2 n_{l}+k+1}\right)=d(A, B)+\epsilon_{0} .
$$

By relation (3.1),

$$
\begin{aligned}
d(A, B)+\epsilon_{0} & \leq d\left(x_{2 m_{l}+2 k}, x_{2 n_{l}+2 k+1}\right) \\
& \leq \alpha^{k} d\left(x_{2 m_{l}+k}, x_{2 n_{l}+k+1}\right)+\left(1-\alpha^{k}\right) d(A, B)
\end{aligned}
$$

Letting $l \rightarrow \infty$ and using (3.5), we obtain

$$
\begin{aligned}
d(A, B)+\epsilon_{0} & \leq \alpha^{k}\left(d(A, B)+\epsilon_{0}\right)+\left(1-\alpha^{k}\right) d(A, B) \\
& =d(A, B)+\alpha^{k} \epsilon_{0} \\
& <d(A, B)+\epsilon_{0}
\end{aligned}
$$

which is a contradiction. Therefore by (3.1) and property $U C_{f},\left\{x_{2 n+k}\right\}$ is a Cauchy sequence by property $U C$. Now the completeness of $X$ and the closedness of $A$ imply that $\lim _{n \rightarrow \infty} x_{2 n+k}=z \in A$, whenever $k$ is a even number. (if $k$ is a odd number, $x_{2 n+k} \rightarrow z \in B$ as $n \rightarrow \infty$.) Since $T$ is a continuous map and $d\left(x_{2 n+k}, x_{2 n+k+1}\right) \rightarrow d(A, B)$ as $n \rightarrow \infty$, hence $d(z, T z)=d(A, B)$. Therefore, $T$ has a best proximity point.

\section{REFERENCES}

[1] A. Abkar, M. Gabeleh, Best proximity points of non-self mappings, Top 21, (2) (2013) 287-295.

[2] M. Ahmadi Baseri and H. Mazaheri, Remotest points and approximate remotest points in metric spaces, Iranian Journal of Science and Technology, Transactions A: Science, (2015) pp.4.

[3] M. A. Al-Thagafi, N. Shahzad, Convergence and existence result for best proximity points, Nonliner Analysis, Theory, Methods and Applications 70, (10) (2009) 3665-3671.

[4] A. Amini-Harandi, Common best proximity points theorems in metric spaces, Optimization Letters, (2014) 581-589.

[5] M. Baronti, A note on remotal sets in Banach spaces, Publication de L'Institute Math. (53) (1993) 95-98.

[6] M. Baronti, P. L. Papini, Remotal sets revisted, Taiwanese J. Math. (5) (2001) 367-373.

[7] A. A. Eldred, P. Veeramani, Existence and convergence of best proximity points, J. Math. Anal. Appl 323, (2) (2006) 1001-1006.

[8] E. Karapinar, Best proximity points of cyclic mappings, Appl. Math. Letters 25, (11) (2012) 1761-1766. 
[9] R. Khalil, Sh. Al-Sharif, Remotal sets in vector valued function spaces, Scientiae Mathematicae Japonicae 63, (3) (2006) 433-442.

[10] W. A. Kirk, P. S. Serinivasan and P. Veeramani, Fixed points for mappings satisfying cyclical contractive conditions, Fixed Point Theory 4, (1) (2003) 79-89.

[11] M. Martín, T. S. S. R. K. Rao, On remotality for convex sets in Banach spaces, J. Approx. Theory. (162) (2010) 392-396.

[12] M. Sababheh, R. Khalil, Remotality of closed bounded convex sets in reflexive spaces, Numer Funct. Anal. Optim, (29) (2008) 1166-1170.

[13] T. D. Narang, A study of farthest points, Nieuw Arch. Wisk. 25 (1977) 54-79.

[14] T. Suzuki, M. Kikkawa and C. Vetro, The existence of best proximity points in metric spaces with the property UC, Nonlinear Analysis: Theory, Methods and Applications 71, (7) (2009) 2918-2926. 